International Journal of Engineering \& Technology, $7(3.20)(2018)$ 334-338
International Journal of Engineering \& Technology
SPC
Website: www.sciencepubco.com/index.php/IJET
Research paper

\title{
Optimization of Material Transportation Assignment for Automated Guided Vehicle (AGV) System
}

\author{
Nor Rashidah Mohamad, "Muhammad Hafidz Fazli Md Fauadi, Siti Fairus Zainudin, \\ Ahamad Zaki Mohamed Noor, Fairul Azni Jafar, Mahasan Mat Ali \\ Faculty of Manufacturing Engineering, Universiti Teknikal Malaysia Melaka, Hang Tuah Jaya, \\ 76100 Durian Tunggal, Melaka, Malaysia \\ *Corresponding Arthur E-mail: hafidz@utem.edu.my
}

\begin{abstract}
:
This article focuses on Material Transportation Assignment problem that is identified as an Automated Guided Vehicles (AGV) multiload task assignment. The primary goal of this paper is to determine the factors needed to optimize material transportation system. This study also explores the optimization and performance enhancement of the Flexible Manufacturing System (FMS) environment. The implementation of Genetic Algorithm (GA) in this model is to obtain the optimal solution for FMS layout. The combination of delivery and pickup task are addressed by modified algorithm for advancement in multiple loads AGV. The result obtained depicts that the proposed task assignment method with a modified genetic algorithm can produce acceptable performance compared to conventional task assignment method.
\end{abstract}

Keywords: Automated Guided Vehicles; Material Transportation Assignment; Genetic Algorithm; FlexSim Software

\section{Introduction}

Automated Guided Vehicle (AGV) system is categorized as a Material Transportation System (MTS) used within a manufacturing floor, warehouses, container terminals and outdoor transportation systems control by a centralized or distributed computer-based control system [15] ; [10]. AGV system is capable to adapt to numerous industrial environments as it possesses flexibility of handling the requirement, reliable in unmanned operation, with medium to high throughput capacity and good in interfacing with dynamic loading requirement. Thus, it is suitable to be used for Flexible Manufacturing System (FMS). Moreover, AGV system becomes intelligent due to the advancement of the industries structure from convenience method to decentralized control.

FMS is defined as manufacturing system which consists of a group of machines connected by an automated material handling system. FMS can rapidly change to allow the process of different products. It is able to handle the uncertainty in product demand knowledge, finite manufacturing capacity, and random machine failures. Practically, simulation becomes handy in providing flexibility of manufacturing system and also the decentralized method of AGVs without experimental on the real time, which can reduce the system risk breakdown, time and cost. Simulation models are of different types that typically depend upon the problem of interest. The main types of simulation modelling techniques used are Agent-Based Simulation, Knowledge-Based Simulation, Discrete Event Simulation and System Dynamics Simulation [3].

Since the primary goal of this study is to determine the factors needed to optimize decentralized material transportation system, discrete event simulation is used to analyze and compare the effectiveness of the method. The secondary goal is to purpose the method to determine material transportation based on the factor required. The relation between the weight of attributes and machine status are difficult to trace by the analytical method. By revising the number of attributes continuously rather than fix it, supply the current status of the effectiveness of production run.

\section{Problem Background}

An AGV is acquired to have functionalities of path planning, scheduling, execution function and decision making. Often this function becomes a possible problem that needs to overcome with an optimal solution. By optimizing the system control approach, it is faster to determine the routes, dissolving the conflicts and collecting information. By encounter the AGV control problems, the system gaining in completed the transportation demand in a fast way with conflict-free among AGVs.

Researchers have given significant attention in studying various AGV related problems, which are task assignment problem, vehicle dispatching problem and vehicle scheduling routing [3] ; [1] ; [8]; [9]; [16]; [14]; [5]. Most of the AGV related problems focusing on the performance of implement system compare with the conventional method and the majority of them are for multicapacity AGV. However, it is becoming more common in factories environment, based on studies [8] ; [9] which identified the implementation of multiple-load AGV can be done by a simple rule and have many advantages.

The industrial environment is a suitable environment to apply a control method with the advancement of the structure by making system feasible in performance, information schedule and computer programming burden. They are important to determine 
the aspects of the AGV system implementation, which are the efficiency of task schedule and routing system of transportation in order to minimize the time movement and avoiding deadlock and collision. Another aspect is the estimation of AGV quantity, required for the tasks assignment [16]. Furthermore, [13] proposed a modified GA to determine the optimal solutions with an acceptable solution of times. This approach employs on crowding distance based to replace the individuals with close proximity. Since the FMS scheduling problem is more complicated, a metaheuristic technique is necessary to solve the scheduling problem.

This study explores the issue of optimization and performance enhancement for the transportation in FMS environment. Therefore, to enhance AGV control problem, the combination of various dispatching rule to a different $A G V$ related problem with multi-capacity AGV are obtained. The research proposes a Genetic Algorithm (GA) to determine the optimal optimization for the FMS layout. Particularly, this approach employs a significant task assignment for AGV to travel along the layout. The participant of AGV to travel and conduct a delivery or pickup task is addressed by modified algorithm following which best solution to the system. The combination of delivery task and pickup task by using different rules among various entities will cause advancement in multiple loads AGV. Therefore, it is important to improve the optimization method in order to solve the problem effectively.

\section{Methodology}

\subsection{Transport Assignment Optimization Model}

In order to gain the optimal solution, AGV task assignment is enhanced with adoptive in GA. Transportation task is acquired to have functionalities which are path planning on the guide path, scheduling the task assignment, execution function and decision making on a network. The FMS layout environment is composed with various workstations and machines; each workstation has a specific continuous operation. Within the FMS, workstations are connected to the guide path network node by pickup/delivery (P/D) point where item is transferred from/to the AGVs and transport between the workstation by the AGVs. Each node is defined as the task assignment $\mathrm{T}_{\mathrm{ij}}$, where $\mathrm{i}$ is the index for job description and $\mathrm{j}$ is the index for processes. The following example depicts the job description:

Job 1, $\mathrm{J}_{1}: \mathrm{T}_{11} \rightarrow \mathrm{T}_{12} \rightarrow \mathrm{T}_{13} \rightarrow \mathrm{T}_{14} \rightarrow \mathrm{T}_{15} \rightarrow \mathrm{T}_{16}$ Job 2, $\mathrm{J}_{2}: \mathrm{T}_{21} \rightarrow \mathrm{T}_{22} \rightarrow \mathrm{T}_{23} \rightarrow \mathrm{T}_{24} \rightarrow \mathrm{T}_{25} \rightarrow \mathrm{T}_{26}$

\subsection{Mathematical Model}

Every AGV is equipped with a mathematical function to enable them to execute a task based on schedule and job sequence. This mathematical function will make the AGV do the combination of multiple tasks simultaneously. The AGV that is adopted to determine the optimal task assignment is based on the integer programming formulation as in Table 1. Meanwhile, variables notation is summarized in Table 2 .

Table 1: Sets of notation and parameter

\begin{tabular}{|l|l|}
\hline Index & Description \\
\hline $\boldsymbol{i} \boldsymbol{j}$ & Set of tasks assignment \\
\hline $\boldsymbol{G}$ & Set of agents \\
\hline $\boldsymbol{X}$ & Set of bids \\
\hline $\boldsymbol{M}$ & Set of AGVs \\
\hline $\boldsymbol{O}$ & Set of machines \\
\hline Index Parameters & Set of operations \\
\hline $\boldsymbol{t} \boldsymbol{d}$ & Delivery time \\
\hline $\boldsymbol{t} \boldsymbol{v}$ & Loading time \\
\hline
\end{tabular}

\begin{tabular}{|c|c|}
\hline $\boldsymbol{t} \boldsymbol{w}$ & Unloading time \\
\hline $\boldsymbol{t} \boldsymbol{p}$ & Travelling time \\
\hline $\boldsymbol{t} \boldsymbol{d}$ & Machine processing time \\
\hline $\boldsymbol{c} \boldsymbol{s}_{\boldsymbol{i}}$ & $\begin{array}{c}\text { Shortest node-to-node distance to transport } \\
\text { load }\end{array}$ \\
\hline $\boldsymbol{t} \boldsymbol{u}$ & Total loading capacity of AGV \\
\hline
\end{tabular}

Consideration of FMS conditions are stated as the following and need to be applied in all case studies that will use this approach. These conditions are including item first arrival at loading area, AGV condition and machine and a network node. Fig. 1 illustrates an example of machines schedule. In our case, AGV scheduling is critical in order to ensure that the machine schedule generated could be achieved.

i) Item flow (or object): All items are starting at the initial time from loading and unloading port.

ii) Guide path routing: The guide path is set as a bidirectional path for the autonomous vehicles.

iii) Dispatching: AGV is set using several types of rules to perform.

iv) Task assignment: AGV transportation tasks are generated by the system yet already assigned to AGVs that can execute them.

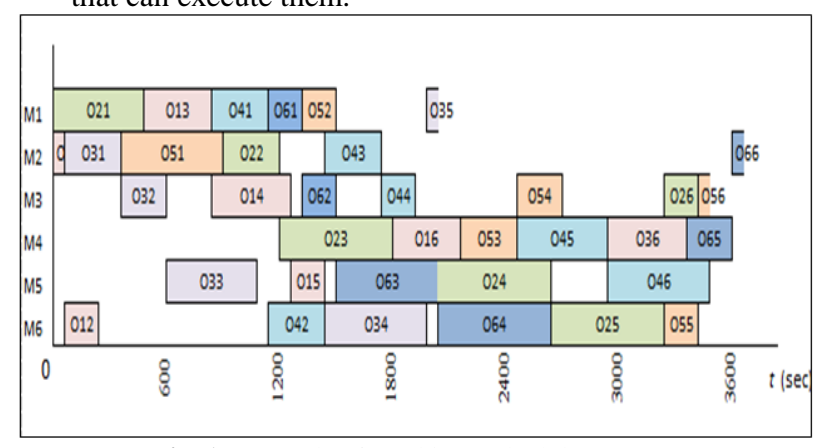

Fig. 1: Example of machines schedule

Table 2: Variables notation

\begin{tabular}{|c|c|}
\hline Variables & Description \\
\hline$a t_{i j}^{m}$ & Expected arrival time of vehicle $m$ for operation $i j$ \\
\hline$t e_{\text {if }}$ & Earliest pickup start time for operation $i j$ \\
\hline$t c_{i f}$ & Starting time of loading of operation \\
\hline$t k_{i f}^{m}$ & Starting time of transportation of operation $i j$ \\
\hline $\operatorname{co}_{m}^{x}$ & Current occupied capacity of AGV $x$ at machine $m$ \\
\hline$c q_{m}^{x}$ & Quantity of pickup/delivery for AGV $x$ at machine $m$ \\
\hline$a d_{i f}^{x}$ & Difference between ${ }^{t e_{i j}}$ and AGVs $a t_{i j}^{m}$ \\
\hline$c n_{x}$ & $\begin{array}{l}\text { Time needed by AGV to complete the transportation } \\
\text { of all loaded tasks. }\end{array}$ \\
\hline$\alpha_{i j}^{m}$ & $\begin{array}{l}\text { Decision variable task assignment which has value } 1 \\
\text { if the operation } i j \text { is assigned to machine } m, 0 \\
\text { otherwise }\end{array}$ \\
\hline$\beta_{\text {if }}^{x}$ & $\begin{array}{l}\text { Decision variable representing status which has value } \\
1 \text { if the operation } i j \text { is assigned to } \mathrm{AGV} x, 0 \text { otherwise }\end{array}$ \\
\hline$\delta_{a b}^{a}$ & $\begin{array}{l}\text { Decision variable which has value } 1 \text { if AGV } x \text { is } \\
\text { travelling from node a to } b, 0 \text { otherwise }\end{array}$ \\
\hline$c e_{m}^{X}$ & $\begin{array}{l}\text { Decision variable representing AGV } x \text { capable to } \\
\text { load and unload at machine } m \text { which has value } 0 \text { if } \\
c 0_{m=c s_{x}^{x}, c 0_{m}^{x}<c s_{x} \text { otherwise }}\end{array}$ \\
\hline
\end{tabular}

The dispatching waiting time for AGV travel to the machine can be minimized and it is translated in the equation (1).

$$
a t_{i j}^{m} \cdot \beta_{i j}^{x}+\sum_{i=1}^{i} \sum_{j=1}^{j} u c \cdot n d \cdot \beta_{i j}^{x}
$$

According to the expected arrival time of vehicle $\mathrm{m}$ for operation ij 


$$
a t_{\mathrm{i} j}^{\mathrm{m}}=t u \cdot c n_{x}
$$

where constraint (2) corresponds the earliest predicted arrival time for the AGV to start the pickup task. The time needed by AGV to complete the transportation of all loaded tasks is computed in equation (3) which defines the expected completion time for the assigned tasks.

$c n_{x}=\sum_{i=1}^{i} \sum_{j=1}^{j}\left[\left(t k_{i j}^{m}+(t d+t w)+(t v+t w)\right) \beta_{i j}^{x}\right]$

Constraint (4) is given by the task assigned to the AGV when estimating the vehicle's expected arrival time. The AGV needs to ensure that the vehicle occupied capacity must be less than total loading capacity of the vehicle given in constraint (5). Equation (6) reflects that the vehicle occupied capacity must travel with non-negative values. Equation (7) represents that the vehicle capacity either pickup or delivery tasks are conducted within the capacity limitation.

$\beta_{i j}^{x}=1$

$$
\begin{aligned}
& c o_{m}^{x}<c s_{x} \\
& c o_{m}^{x} \geq 0 \\
& c o_{m}^{x}+c q_{m}^{x} \leq \sum_{m \in S}^{s} \delta_{a b}^{x} \cdot c s_{x}
\end{aligned}
$$

The developed mathematical model is then fitted as the GA fitness function to optimize the AGV scheduling problem. In this case, the problem is treated as a constrained optimization problem. Standard GA is utilized where each of the possible solutions are treated as an individual in the search population. In each of the iteration, next set of candidate solutions are generated by several operators including mutation, crossover and reproduction. One of the reasons why GA is selected is due to the reason that the FMS consists the elements of combinatorial problem. Using GA to optimize the problem is suitable as it is capable to significantly reduce the computational time. Fig. 2 depicts the example of crossover and mutation. This article does not include the comparison of GA against other optimization methods. However, lengthy discussions and comparisons have been made by [2], [7], [12] and [11].

\begin{tabular}{|l|l|l|l|l|l|}
\hline 2 & 3 & 5 & 4 & 6 & 3 \\
\hline 1 & 2 & 4 & 6 & 3 & 5
\end{tabular}$\longrightarrow$

Selection

\begin{tabular}{|l|l|l|l|l|l|}
\hline 1 & 2 & 4 & 4 & 6 & 1 \\
\hline 2 & 3 & 5 & 6 & 3 & 5 \\
\hline
\end{tabular}

Crossover

Fig. 2 Example of GA crossover and mutation

\begin{tabular}{|l|l|l|l|l|l|}
\hline 1 & 2 & 4 & 4 & 6 & 1 \\
\hline 2 & 3 & 5 & 6 & 3 & 5 \\
\hline
\end{tabular}

\begin{tabular}{|l|l|l|l|l|l|}
\hline 1 & 2 & 4 & 5 & 6 & 3 \\
\hline 2 & 3 & 5 & 6 & 1 & 4 \\
\hline
\end{tabular}

\section{Simulation Experiment}

The simulation models are set as the routing system simulates routes for the AGVs, it calculates conflict-free route at a minimized cost, and provides the total time spent and the total time estimated for the execution of all tasks assigned to each AGV. The AGV agent needs to have several main functionalities in the system that need to perform transport assignment, routing, collision avoidance and deadlock avoidance. The main functionalities are discussed below: The criteria of the case are summarized as follows:

- The layout consists of six CNC machines, three AGVs and a dispatcher as a controller.

- Consider the AGVs capable on multiple-load task.

- Each workstation has delivery and pickup point is arranged in a way so that when an AGV is approaching a workstation, it will reach the workstation's delivery point first.

- The number of AGVs in the system is set by numerical indication.

- The layout of workstation and path were determined. The layout is depicted in Fig. 3

- Idle AGVs will stay at standby location to wait for delivery and pickup requests from workstations.

- Loading and unloading times are fixed at 30 seconds each.

- A vehicle travels in a bi-directional guide path with a constant velocity of $40 \mathrm{~m} / \mathrm{min}$.

This model simulation is conducted using FlexSim Software discrete event simulation; by evaluating neither the task given in combination delivery nor dispatch task and purpose approach. With this, purpose system is employed to obtain the performance measurement of different aspects of the mechanism by using three performance indicators.

\section{Result And Discussion}

Performance Measurements of MTS in a Manufacturing Industry called as Performance indicators (PIs) are typically utilized to measure the

successfulness of a particular proposed method to achieve stated objectives. This result section only illustrates the preliminary result in the early stage. It is important to select appropriate PIs so as to measure critical aspects resulted from the implemented proposal. Some of the PIs used in this research are categorized as the following:

- System throughput (STH) refers to the number of finished unit produced within a specific time period. The result shown in Fig. 4 ascertains that multi-load AGVs on achieving the throughput. The box plot shows the capability of different fleet size during the simulation going. The more vehicles are using the less single fleet work load. Additionally, during the deployment of simulation, the best solution is using 3 vehicles in this experimentation compared to 4 and more vehicles which the throughput result is similar.

- Meanwhile, Fig. 5 illustrates the conventional method compare to proposed method. The achievement of propose method lowering the convenience method shown in the graft. In this simulation, a conventional method is referred to transportation task assignment approach that using a heuristic method.

- $\quad$ Average pickup waiting time (AWT) that measures the time difference between actual vehicle arrival times and the earliest pickup time. The result in Fig. 6 illustrates the average pickup waiting times that reduce comparing using the convenience method. Meanwhile, in Fig. 7 the 
graph is comparing transportation completed job between proposed approach and a conventional method.

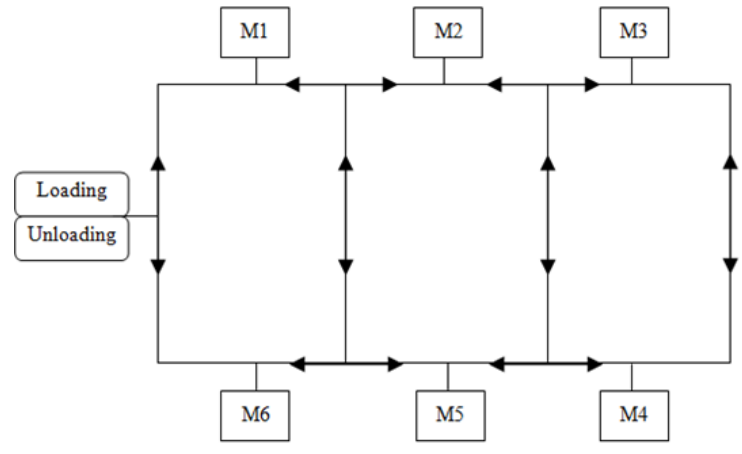

Fig. 3: FMS Layout

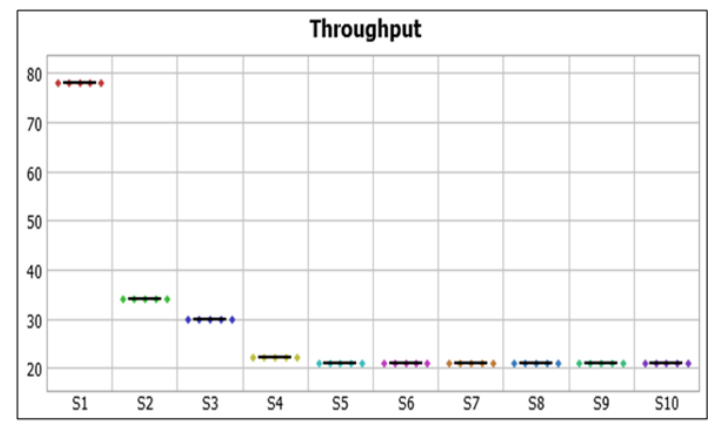

Fig. 4: Throughput/ vehicle for different fleet size

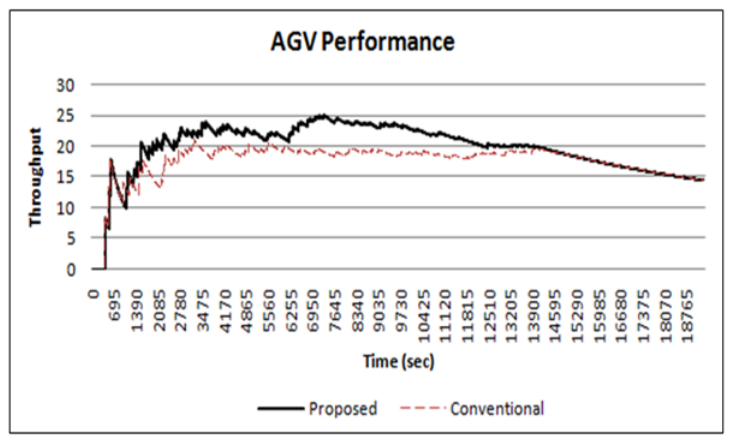

Fig. 5 AGV Performance (Throughput)

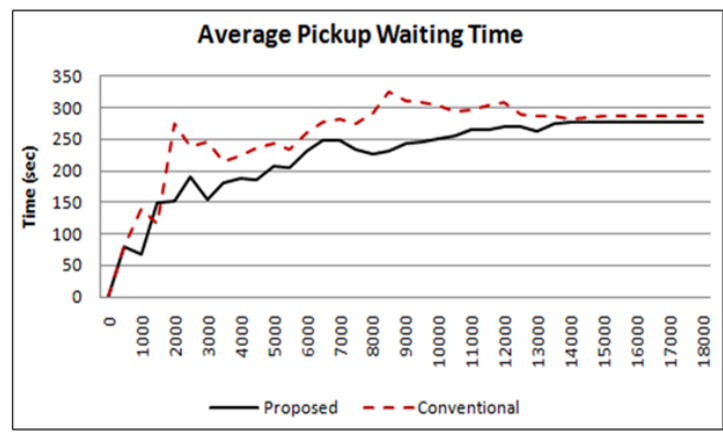

Fig. 6: AGV Performance (Pickup Waiting Time)

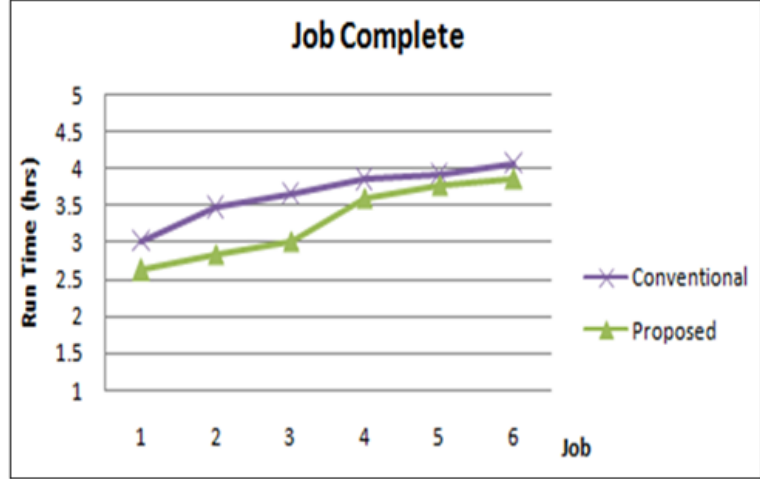

Fig. 7: Completion time

This paper proposed a GA with dispatching rule to implement. In order to perform with the effectiveness of GA consideration of machine distance, dispatch waiting time is needed. Consideration of delivery and pickup task are important to obtain an initial result. Although the critical values between the vehicles are not the same, these purpose methods perform competitive outcome compared to the conventional approach. Moreover, it also shows a performance in task assignment approach significantly in reducing total vehicle travel.

\section{Acknowledgement}

The authors wish to thank Malaysian Ministry of Education for financial support (FRGS/1/2014/TK01/FKP/02/F00218) and Universiti Teknikal Malaysia Melaka (UTeM).

\section{References}

[1] Ahmad H. and Othman N. A. "HF-Fuzzy logic based mobile robot navigation: A solution to finite escape time", ARPN Journal of Engineering and Applied Sciences Vol. 10 No. 23, December 2015.

[2] Cochran J. K., Horng, S. M., \& Fowler, J. W. (2003). A multipopulation genetic algorithm to solve multi-objective scheduling problems for parallel machines. Computers \& Operations Research, 30(7), 1087-1102.

[3] Fauadi M.H.F.M., Yahaya S.H., Murata T., 2013. Intelligent combinatorial auctions of decentralized task assignment for AGV with multiple loading capacity, IEEJ Transactions on Electrical and Electronic Engineering, 8(4):371-379.

[4] Fauadi M.H.F.M., Lin H., Murata T, 2010. Dynamic task assignment of autonomous AGV system based on multi agent architecture, Progress in Informatics and Computing (PIC), IEEE International Conference, pp 1151-1156.

[5] Fauadi M.H.F.M., Murata T., 2010. Makespan Minimization of Machines and Automated Guided Vehicles Schedule Using Binary Particle Swarm Optimization. Proceedings of the International MultiConference of Engineers and Computer Scientists (IMECS) Volume 3 pp. 1897-1902

[6] Fauadi, M. H. F. B. M., Li, W. L., \& Murata, T., 2011. Combinatorial auction method for decentralized task assignment of multiple-loading capacity AGV based on intelligent agent architecture. In Innovations in Bio-inspired Computing and Applications (IBICA), 2011 Second International Conference on (pp. 207-211). IEEE.

[7] Gandomi, A. H. et al (Eds.). (2013). Metaheuristic applications in structures and infrastructures. Newnes.

[8] Ho Y.C., Chien S.H., 2006. A simulation study on the performance of delivery dispatching rules for multiple-load AGVs, International Journal of Production Research, 44(20):4193-4222.

[9] Ho Y.C., Liu H.C., 2006. A simulation study on the performance of pickup-dispatching rules for multiple-load AGVs", Computers and Industrial Engineering, 51(3):445-463.

[10] Le-Anh T., De Koster, M.B.M., "A review of design and control of automated guided vehicle systems", European Journal of Operational Research, 171(1):1-23, 2006 
[11] Nguyen, A. T., Reiter, S., \& Rigo, P. (2014). A review on simulation-based optimization methods applied to building performance analysis. Applied Energy, 113, 1043-1058.

[12] Parejo, J. A., Ruiz-Cortés, A., Lozano, S., \& Fernandez, P. (2012). Metaheuristic optimization frameworks: a survey and benchmarking. Soft Computing, 16(3), 527-561.

[13] Rifai A.P., Dawal S.Z.M., Zuhdi A., Aoyama H., Case K.. "Reentrant FMS scheduling in loop layout with consideration of multi loading-unloading stations and shortcuts", International Journal of Advance Manufacturing Technology, Vol. 82, Issue 9, pp 1527-1545, 2016

[14] Sulle B., Adriansyah A, and Dewi S. S.. "Coordination of mobilerobot system with behavior-based architecture", ARPN Journal of Engineering and Applied Sciences. Vol. 10, No. 16, September 2015

[15] Vis I. F. A.., "Survey of research in the design and control of automated guided vehicle systems", European Journal of Operational Research, 170(3): 677-709, 2006

[16] Vivaldini K.C.T. et al, "Integrated tasks assignment and routing for the estimation of the optimal number of AGVS", International Journal of Advance Manufacturing Technology, Vol. 82, Issue 1, pp 719-736, 2016. 